\title{
Safety of regadenoson with theophylline reversal during dynamic computed tomography perfusion and magnetic resonance imaging in patients with coronary artery disease
}

\author{
Anna Oleksiak1, Mariusz Kruk², Mateusz Śpiewak³, Barbara Miłosz-Wieczorek³, \\ Magdalena Marczak³ ${ }^{3}$ Marcin Demkow², Cezary Kępka² \\ 1 Department of Intensive Cardiac Therapy, National Institute of Cardiology, Warsaw, Poland \\ 2 Department of Coronary and Structural Heart Diseases, National Institute of Cardiology, Warsaw, Poland \\ 3 Magnetic Resonance Unit, Department of Radiology, National Institute of Cardiology, Warsaw, Poland
}

\section{KEY WORDS}

computed tomography perfusion, coronary artery disease, magnetic resonance myocardial perfusion imaging, regadenoson, theophylline

\section{Correspondence to:}

Anna Oleksiak, MD, PhD, Department of Intensive Cardiac Therapy National Institute of Cardiology, ul. Alpejska 42, 04-628 Warszawa, Poland, phone: +48223434314 , email: aoleksiak@ikard.pl Received: February 28, 2020. Revision accepted: May 8, 2020. Published online: May 12, 2020. Kardiol Pol. 2020; 78 (7-8): 709-714 doi:10.33963/KP.15356 Copyright by the Author(s), 2020

\section{ABSTRACT}

BACKGROUND The use of regadenoson in dynamic computed tomography perfusion (CTP) and magnetic resonance myocardial perfusion imaging (MR MPI) is off-label.

AIMS The study aimed to assess the safety of regadenoson with theophylline reversal during CTP and MR MPI in patients with coronary artery disease (CAD).

METHODS In this prospective study, patients with 1 or more intermediate coronary artery stenoses on computed tomography angiography underwent CTP and MR MPI with $0.4 \mathrm{mg}$ of regadenoson. After examinations, $200 \mathrm{mg}$ of theophylline was given intravenously in $100 \mathrm{ml}$ of saline. Changes in blood pressure (BP) and heart rate (HR) were repeatedly assessed. All side effects and adverse events were recorded.

RESULTS Out of 106 examinations in 53 patients ( 25 females, 63.5 [8.5] years), all were diagnostic. There were no deaths, myocardial infarctions, severe arrhythmias, high-grade atrioventricular blocks, or bronchospasms. The most common symptoms were palpitations (17\%), hot flushing (8\%), chest discomfort (4\%), and mild dyspnea (3\%). There were no differences between baseline and peak BP. There was an increase in median (interquartile range) peak $\mathrm{HR}$ after regadenoson as compared with baseline (MR MPI, 63 [59-75] bpm vs 93 [86-102] bpm; $P<0.001$; and CTP, 65 [60-70] bpm vs 95 [86-107] bpm; $P<0.001)$. The hemodynamic response to regadenoson and its side effects were completely reversible by theophylline. CONCLUSIONS Regadenoson may be a safe vasodilator for CTP and MR MPI in patients with CAD. The administration of theophylline after perfusion is safe and reverses side effects of regadenoson.

INTRODUCTION Vasodilating stress agents are administrated to induce vasodilation of the coronary microcirculation. The European Medicines Agency and the United States Food and Drug Administration approved 3 vasodilators for myocardial perfusion imaging: regadenoson, adenosine, and dipyridamole. The use of regadenoson in dynamic computed tomography perfusion (CTP) and magnetic resonance myocardial perfusion imaging (MR MPI) is considered off-label because this agent was registered only for radionuclide perfusion imaging (scintigraphy) and invasive fractional flow reserve measurements.

Vasodilators bind to several adenosine receptors $\left(A_{1}, A_{2 A}, A_{2 B}\right.$, and $\left.A_{3}\right)$ located in different tissues, which control various physiological functions. ${ }^{1}$ However, mainly the stimulation of $\mathrm{A}_{2 \mathrm{~A}}$ receptors provokes coronary vasodilation, and 


\section{WHAT'S NEW?}

The use of regadenoson as a pharmacologic stressor in dynamic computed tomography perfusion and magnetic resonance myocardial perfusion imaging is off-label as yet; however, there is a need for this drug approval for these imaging modalities. Our study showed that regadenoson with theophylline reversal may be a safe drug combination for dynamic computed tomography perfusion and myocardial perfusion imaging in patients with coronary artery disease.

the stimulation of other types of receptors may be associated with severe side effects such as atrioventricular block ( $A_{1}$ receptors) or bronchoconstriction ( $\mathrm{A}_{2 \mathrm{~B}}$ and $\mathrm{A}_{3}$ receptors). ${ }^{1}$ Regadenoson, as the most selectively acting vasodilator, has a 9-fold stronger affinity for $\mathrm{A}_{2 \mathrm{~A}}$ receptors than adenosine. ${ }^{2-4}$ Their affinity for other types of adenosine receptors is residual..$^{2-4}$ Moreover, regadenoson has been shown to be noninferior to adenosine and has fewer side-effects in several nuclear imaging trials. ${ }^{5-7}$ As it has been described previously, most patients receiving regadenoson had mild and transient side-effects; however, the Food and Drug Administration reported some serious adverse events even including death and nonfatal cardiac arrest. . $^{5,9}$

The effects of vasodilators could be reversed by aminophylline, containing theophylline, and ethylenediamine. Aminophylline is a nonselective antagonist of adenosine receptors. However, only $3 \%$ of patients received it to reverse the effect of regadenoson in the ADVANCE-MPI (Adenosine Versus Regadenoson Comparative Evaluation in Myocardial Perfusion Imaging) trial. ${ }^{5}$ As reported by Doukky et al, ${ }^{6}$ patients had greater satisfaction with regadenoson stress testing with routine reversal by aminophylline. Due to limited availability of aminophylline, theophylline may prove to be an effective alternative to reverse side effects of regadenoson. Data regarding theophylline reversal are still lacking.

Knowledge of adverse events associated with regadenoson dynamic $C T P$ and MR MPI has implications for both patients and staff. Thus, we sought to evaluate the safety of regadenoson with theophylline reversal for these perfusion imaging modalities in patients with coronary artery disease (CAD), who are more susceptible to ischemia-induced arrhythmias, conduction abnormalities, or angina during functional testing.

METHODS Study population Out of patients who underwent coronary computed tomography angiography (CTA) for suspected CAD, those with 1 or more intermediate $(50 \%-90 \%)$ coronary artery stenoses were recruited. The exclusion criteria were: history of myocardial infarction, symptoms of unstable angina or acute coronary syndrome, history of revascularization (percutaneous coronary intervention or coronary artery bypass grafting), impaired renal function (estimated glomerular filtration rate $<60 \mathrm{ml} / \mathrm{min} / 1.72 \mathrm{~m}^{2}$ ), contraindications to computed tomography or magnetic resonance (including pregnancy, claustrophobia, implanted cardiac device, implanted metal elements, etc), contraindications to the administration of an iodine contrast media (including allergy to the contrast agent, unstable hyperthyroidism, etc) or regadenoson (hypersensitivity to the active substance, unstable angina, second- or third- degree atrioventricular block, sinus node dysfunction, hypotension, decompensated heart failure, etc), persistent atrial fibrillation or flutter, heart failure with reduced ejection fraction, a significant valvular heart disease, aortic dissection or aortic aneurysm, hypertrophic cardiomyopathy, epilepsy, and previous stroke.

The study protocol received approval from the ethics committee and all patients gave written informed consent. The ClinicalTrials.gov identifier is NCT03917199.

\section{Examination protocols and safety monitoring} All recruited patients underwent dynamic CTP using a dual-source computed tomography scanner (Somatom Force, Siemens, Germany) and MR MPI (AvantoFIT, Siemens, Germany) in a median (interquartile range [IQR]) 2 (1.4-3.1) weeks' interval according to the study protocol. ${ }^{10}$ Patients were asked to refrain from caffeine, theophylline, and $\beta$-blockers for 24 hours before the stress tests. In both perfusion examinations, regadenoson at a single dose of $0.4 \mathrm{mg}$ was given intravenously in 5 to 10 second bolus, followed by $20 \mathrm{ml}$ of saline. After the stress test, $200 \mathrm{mg}$ of theophylline in $100 \mathrm{ml}$ of saline was administered intravenously.

Electrocardiogram (ECG), blood pressure (BP), and heart rate (HR) were monitored before, during, and after the exam. Peak HR was defined as the highest HR during the stress perfusion scan and prior to the administration of theophylline. Peak BP was defined as the BP prior to reversal with theophylline. Regadenoson-induced tachycardia was defined as HR greater than $100 \mathrm{bpm}$. Hypotension was defined as systolic blood pressure (SBP) of less than $90 \mathrm{~mm} \mathrm{Hg}$.

Patients were questioned about their symptoms before and after regadenoson and after theophylline administration. Stress-related adverse events including death, myocardial infarction, severe ventricular or supraventricular arrhythmias, high-grade atrioventricular block, and bronchospasm requiring medical treatment were assessed. Other adverse events, including contrast extravasation or reaction to gadolinium or iohexol were registered.

Magnetic resonance myocardial perfusion imaging protocol Patients were examined with a $1.5 \mathrm{~T}$ MR scanner (AvantoFIT, Siemens, Germany). After regadenoson infusion, $0.1 \mathrm{mmol} / \mathrm{kg}$ of 
gadobutrol (Gadovist 1.0, Bayer Pharma AD) was infused at $3.5 \mathrm{ml} / \mathrm{s}$ flow rate and followed by $20 \mathrm{ml}$ of saline. Stress-perfusion acquisition of the left ventricle with first-pass perfusion technique at 3 short-axis views, basal, mid-ventricular, and apical, were performed. 10 minutes after the infusion of contrast agent, a contrast enhanced segmented T1-weighted inversion-recovery gradient-echo sequence was acquired to detect late gadolinium enhancement, followed by rest first-pass perfusion imaging at the end of the examination.

Computed tomography perfusion protocol The scan range was determined based on a low-dose noncontrast scan. Fifty seconds after regadenoson administration, $35 \mathrm{ml}$ of iodinated

TABLE 1 Baseline patient characteristics

\begin{tabular}{|c|c|}
\hline Parameter & Value $(n=53)$ \\
\hline Age, y, mean (SD) & $63.5(8.5)$ \\
\hline Female sex & $25(47)$ \\
\hline BMI, $\mathrm{kg} / \mathrm{m}^{2}$, mean (SD) & $27.7(3.6)$ \\
\hline \multicolumn{2}{|l|}{ CAD risk factors } \\
\hline Hypertension & $46(87)$ \\
\hline Dyslipidemia & $52(98)$ \\
\hline Diabetes & $13(25)$ \\
\hline Family history & $31(58)$ \\
\hline Smoking (current) & $5(9)$ \\
\hline Smoking (past) & $26(49)$ \\
\hline \multicolumn{2}{|l|}{ Medications } \\
\hline$\beta$-Blocker & $39(74)$ \\
\hline Statin & $42(79)$ \\
\hline PPARa agonist & $4(8)$ \\
\hline ACEI & $20(38)$ \\
\hline ARB & $17(32)$ \\
\hline Calcium antagonist & $21(40)$ \\
\hline Diuretic & $12(23)$ \\
\hline Metformin & $13(25)$ \\
\hline Acetylsalicylic acid & $43(81)$ \\
\hline \multicolumn{2}{|l|}{ Coronary CTA results } \\
\hline CASC (Agatston), median (IQR) & $366.7(109.2-727.6)$ \\
\hline 3-vessel CAD & 0 \\
\hline 2-vessel CAD & $15(28)$ \\
\hline 1-vessel CAD & $38(72)$ \\
\hline
\end{tabular}

Data are presented as number (percentage) unless otherwise indicated.

Abbreviations: ACEI, angiotensin converting enzyme inhibitor; ARB, angiotensin receptor blocker; $B M I$, body mass index; $C A D$, coronary artery disease; $C A S C$, coronary artery calcium score; CTA, computed tomography angiography; PPARa, peroxisome proliferator-activated receptor a contrast agent (iohexol, $350 \mathrm{mg}$ iodine $/ \mathrm{ml}$ ) was infused at the flow rate of $5 \mathrm{ml} / \mathrm{s}$, followed by $20 \mathrm{ml}$ of saline. The scan parameters were: collimation of $2 \times 192 \times 0.6 \mathrm{~mm}$, gantry rotation time of $250 \mathrm{~ms}$, temporal resolution of $66 \mathrm{~ms}$, fixed tube voltage of $70 \mathrm{kV}$, automatic tube current up to $400 \mathrm{mAs}$, as described previously. ${ }^{10}$

Statistical analysis Continuous variables were presented as median with interquartile range (IQR) or mean (SD). Categorical variables were presented as percentages or frequencies. Differences between the quantitative variables were determined for normal distribution by the $t$ tests, for nonnormal distribution by the Mann-Whitney test (independent variables) or the Wilcoxon test (paired variables). Differences between qualitative variables were determined using the $\mathrm{X}^{2}$ test. A $P$ value of less than 0.05 was considered statistically significant. The analyses were performed using MedCalc version 18.11.3 (MedCalc Software, Ostend, Belgium).

RESULTS Study population Out of $53 \mathrm{pa}$ tients (25 females; mean [SD] age, 63.5 [8.5] years) who underwent both perfusion examinations, 15 (27\%) were diagnosed with reversible myocardial ischemia by MR MPI. Baseline characteristics of the study population and main computed tomography angiography findings are presented in TABLE 1 according to the data described previously. ${ }^{10}$

Electrocardiographic changes In 12-lead rest ECG, 3 patients had first-degree atrioventricular block. No other signs of arrhythmia were detected by 12-lead rest ECG. During perfusion examinations, there were no severe ventricular and supraventricular arrhythmias or high-grade (2nd or 3rd degree) atrioventricular block observed, even in patients with preexisting first-degree atrioventricular block. None of the patients developed atrioventricular block.

Hemodynamic response to regadenoson and theophylline Regadenoson-induced tachycardia occurred in 16 patients during CTP and 16 patients during MR MPI. There was an increase in median (IQR) peak HR after regadenoson as compared with baseline (MR MPI, 63 [59-75] bpm vs 92.5 [86-102] bpm respectively; $P<0.001$ and CTP, 65 [60-70] bpm vs 95 [86-107] bpm respectively; $P<0.001$ ) (FIGURE 1). The median (IQR) increase in HR after the administration of regadenoson was 29 (22-34) bpm during MR MPI and 30 (22-38) bpm during CTP $(P=0.4)$. Median (IQR) HR decreased after intravenous theophylline administration (MR MPI, 66 [60-76] bpm vs peak value; $P<0.001$, and CTP, 68 [62-75] bpm vs peak value; $P<0.001$ ) (figure 1). An increase in HR of $20 \%$ 


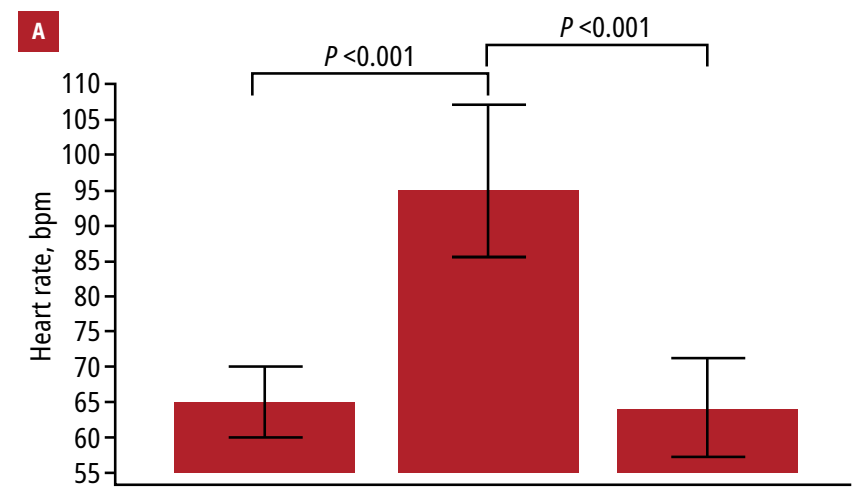

Before regadenoson After regadenson After theophylline

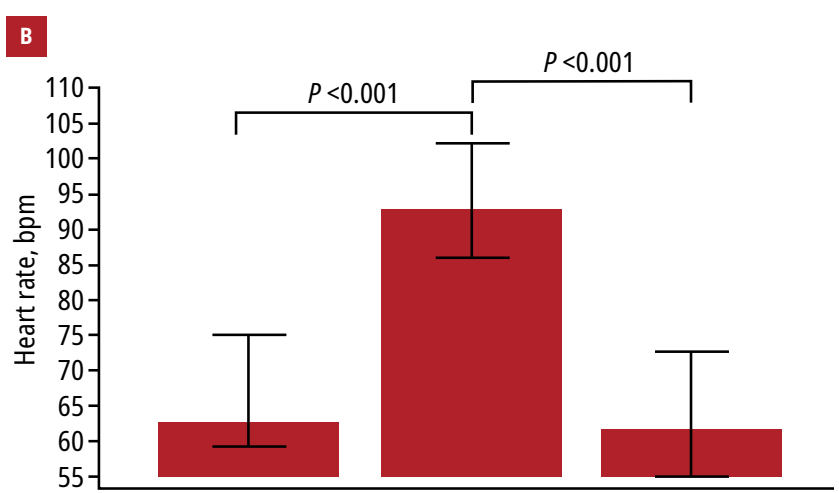

Before regadenoson After regadenson After theophylline

FIGURE 1 Heart rate before and after regadenoson administration during computed tomography perfusion (A) and magnetic resonance myocardial perfusion (B). Whiskers represent interquartile ranges for medians.

TABLE 2 Blood pressure before examination, peak value after regadenoson administration and after theophylline reversal during computed tomography perfusion and magnetic resonance myocardial perfusion

\begin{tabular}{|c|c|c|c|c|c|}
\hline Parameter & Before regadenoson & After regadenoson & $P$ value & After theophylline & $P$ value \\
\hline \multicolumn{6}{|l|}{ MR MPI } \\
\hline SBP, mm Hg & $128(17)$ & $128(18)$ & 1 & $125(14)$ & 0.06 \\
\hline $\mathrm{DBP}, \mathrm{mm} \mathrm{Hg}$ & $73(10)$ & $74(13)$ & 0.5 & $72(9)$ & 0.5 \\
\hline \multicolumn{6}{|l|}{ СTP } \\
\hline SBP, mm Hg & $134(21)$ & $130(16)$ & 0.03 & $134(16)$ & 0.1 \\
\hline $\mathrm{DBP}, \mathrm{mm} \mathrm{Hg}$ & $79(8)$ & $76(9)$ & 0.06 & $80(10)$ & 0.04 \\
\hline
\end{tabular}

Data are presented as mean (SD).

a $P$ value before and after regadenoson

b $P$ value before and after theophylline

Abbreviations: CTP, computed tomography perfusion; DBP, diastolic blood pressure; MR MPI, magnetic resonance myocardial perfusion imaging; SBP, systolic blood pressure

TABLE 3 Symptoms reported by patients during examinations

\begin{tabular}{lll} 
Symptoms & CTP & MR MPI \\
Palpitations & 11 & 8 \\
\hline Hot flushing & 3 & 6 \\
\hline Chest discomfort & 2 & 3 \\
\hline Mild dyspnea & 0 & 3 \\
\hline Headache & 0 & 2 \\
\hline Nausea & 0 & 1 \\
\hline Tremor & 1 & 0 \\
\hline Facial redness & 1 & 0 \\
\hline Metallic taste & 1 & 0 \\
\hline Allergic reaction & 0 & 1 \\
\hline
\end{tabular}

Data are presented as the number of patients.

Abbreviations: See TABLE2 or higher from baseline was reached in all perfusion examinations, of $30 \%$ or higher from baseline in three-fourths of perfusion examinations, and of $50 \%$ or higher from baseline in one-third of perfusion examinations. In all examinations $(\mathrm{n}=112)$, the increase in HR did not depend on sex $(P=0.2)$, age $(P=0.3)$, ischemia $(P=0.06)$, or CAD risk factors: hypertension $(P=0.7)$, diabetes $(P=0.7)$, family history of $\operatorname{CAD}(P=0.5)$, and smoking $(P=0.3)$.

None of the patients had hypotension. Systolic and diastolic blood pressure (DBP) before and after regadenoson administration during CTP and MR MPI are given in TABLE2. In MR MPI, SBP and DBP did not differ before and after regadenoson administration $(P=1$ and $P=0.5$, respectively) as well as before and after theophylline administration $(P=0.06 ; P=0.5$, respectively). In CTP, SBP was significantly lower after regadenoson administration $(P=0.03)$, but did not differ before and after theophylline administration $(P=0.1)$. DBP did not differ before and after regadenoson administration $(P=0.06)$, but was higher after theophylline administration $(P=0.04)$. 
Adverse events Overall, there were few minor adverse events registered. Palpitations, hot flushing, chest discomfort, and mild dyspnea were the 4 most frequently reported symptoms (TABLE3). There were no side effects observed after theophylline administration. One patient with tremor did not receive theophylline. In this case, tremors were mild and stopped after a few minutes of clinical observation. All other reported regadenoson side effects were completely reversible during or immediately after theophylline administration.

One patient had an allergic reaction to the contrast agent (gadobutrol) used in MR MPI and required hospitalization. We observed no other adverse events.

DISCUSSION Our study shows that regadenoson dynamic CTP and MR MPI could be performed with mild side effects and theophylline reversal after regadenoson administration is safe and effective. The use of regadenoson in pharmacologic stress CTP and MR MPI is off-label; however, there is a need for this drug approval for these imaging modalities. Previously, 2 studies reported on the administration of regadenoson in static CTP and dynamic CTP; however, the second study did not report adverse events of regadenoson..$^{11,12}$ Cury et a ${ }^{11}$ showed that $69 \%$ of patients had adverse effects after the administration of regadenoson, and $43.2 \%$ of these were considered mild; however, only 1 patient received aminophylline for symptoms reversal. Only one retrospective study reported safety of regadenoson MR MPI and showed that $47.4 \%$ of patients reported symptoms, but none of them were serious adverse events. ${ }^{13}$

In our study, only mild and transient symptoms not requiring medical intervention were observed. The reported mild symptoms were comparable to those observed in other studies. The most common side effects reported during clinical trials were dyspnea (29\%), headache (27\%), flushing (23\%), chest pain (19\%), ST-segment changes on ECG (18\%), gastrointestinal discomfort (15\%), and dizziness (11\%). ${ }^{8}$ Townsend et $\mathrm{al}^{9}$ in a study performed on healthy volunteers showed that after administration of regadenoson, the most commonly reported symptoms were headache, nausea, dizziness, and sweating. Doran et $\mathrm{al}^{14}$ in the SPECT study reported dyspnea (68\%), gastrointestinal discomfort (36.5\%), headache (33.6\%), and flushing (32.8) as the 4 most common symptoms. In our study, there were no deaths, myocardial infarctions, severe ventricular or supraventricular arrhythmias, bronchospasms or high-grade atrioventricular blocks.

We observed decrease in SBP after regadenoson administration during CTP $(P=0.03)$, but there were no differences in DBP. The median (IQR) increase in $\mathrm{HR}$ after administration of regadenoson was 29 (22-34) bpm during MR MPI and 30 (22-38) bpm during CTP. In the ADVANCE-MPI trials, the 2 most common cardiovascular side effects following the administration of regadenoson included modest reductions in SBP and DBP (average of 13 and $8 \mathrm{~mm} \mathrm{Hg}$, respectively) and also a modest increase in HR (mean [SD] increase of 25 [11] bpm), similarly to observed in our study. ${ }^{7}$

Based on the search of available literature, special care should be taken in patients with a history of epilepsy or seizures, as there are a few reports indicating that regadenoson may cause seizures or tremors, especially in predisposed patients. ${ }^{15,16}$ In case of this type of adverse reaction, aminophylline (or theophylline) should not be administered because there is a possibility of prolonged seizures. ${ }^{15,16}$ In our study, one patient with tremor did not receive theophylline.

The uniqueness of regadenoson as a vasodilator is associated with: 1) high selectivity for adenosine $A_{2 A}$ receptors resulting in a more favorable patient safety profile compared with adenosine and dipyridamole (due to their nonselectivity, both adenosine and dipyridamole cause more side effects); 2) no contraindication for patients with asthma and chronic obstructive pulmonary disease; 3) ease and convenience of administration (5-10 second bolus administered to the peripheral vein); 4) no need to adjust the dose to the patient's age, sex, weight, kidney and liver function; and 5) regadenoson causes only a mild decrease of renal blood flow (compared with adenosine, which caused renal vasoconstriction-increase of vascular resistance and significant decrease of renal blood flow). ${ }^{5,17-20}$ It is also worth emphasizing that CTP with adenosine requires 2 intravenous accesses (separate continuous adenosine infusion and continuous contrast agent infusion), whereas in the case of CTP with regadenoson only one intravenous access is necessary. This affects the comfort both of the patient and the nursing team responsible for obtaining vascular access. Considering the above advantages and safety, regadenoson seems to be an optimal vasodilator for functional testing, successfully reversible by theophylline.

Limitations This is a single-center study, therefore, the study sample was relatively small and applicability of those findings to a more general population may not be straightforward.

We did not monitor 12-lead ECG ST-T changes during perfusion, only continuous monitoring for arrhythmia detection was applied.

Based on product characteristics, during dynamic CTP, the following symptoms could be associated with both regadenoson and iodine contrast agent: headache, feeling hot, metallic taste in the mouth. During MR MPI, the following symptoms could be associated with 
both regadenoson and contrast agent: headache, nausea, feeling hot, shortness of breath, and palpitations.

All patients underwent 2 examinations with regadenoson. Most of reported symptoms occurred in only the first performed modality. It is not known whether second administration of regadenoson affected the severity of symptoms; however, we maintained an adequate interval between these examinations.

Conclusions Regadenoson may be a safe vasodilator for dynamic CTP and MR MPI in patients with obstructive CAD and was associated with mild side effects in this study. Theophylline administered after perfusion examinations is safe and reverses regadenoson-induced side effects.

\section{ARTICLE INFORMATION}

ACKNOWLEDGMENTS This study was funded by the National Science Centre, Poland (no. 2015/19/B/NZ5/03502; to CK).

CONFLICT OF INTEREST None declared.

OPEN ACCESS This is an Open Access article distributed under the terms of the Creative Commons Attribution-Non Commercial-No Derivatives 4.0 International License (CC BY-NC-ND 4.0), allowing third parties to download articles and share them with others, provided the original work is properly cited, not changed in any way, distributed under the same license, and used for noncommercial purposes only. For commercial use, please contact the journal office at kardiologiapolska@ptkardio.pl.

HOW TO CITE Oleksiak A, Kruk M, Śpiewak M, et al. Safety of regadenoson with theophylline reversal during dynamic computed tomography perfusion and magnetic resonance imaging in patients with coronary artery disease. Kardiol Pol. 2020; 78: 709-714. doi:10.33963/KP.15356

\section{REFERENCES}

1 Borea PA, Gessi S, Merighi S, et al. Pharmacology of adenosine receptors: the state of the art. Physiol Rev. 2018; 98: 1591-1625.

2 Gao Z, Li Z, Baker SP, et al. Novel short-acting $A_{2 A}$ adenosine receptor agonists for coronary vasodilation: inverse relationship between affinity and duration of action of A2A agonists. J Pharmacol Exp Ther. 2001; 298: 209-218.

3 Andrikopoulou E, Hage FG. Adverse effects associated with regadenoson myocardial perfusion imaging. J Nucl Cardiol. 2018; 25: 1724-1731.

4 Al Jaroudi WA, Iskandrian AE. Regadenoson: a new myocardial stress agent. J Am Coll Cardiol. 2009; 54: 1123-1130.

5 Cerqueira MD, Nguyen P, Staehr P, et al. Effects of age, gender, obesity, and diabetes on the efficacy and safety of the selective $A_{2 A}$ agonist regadenoson versus adenosine in myocardial perfusion imaging integrated ADVANCE-MPI trial results. JACC Cardiovasc Imaging. 2008; 1: 307-316.

6 Doukky R, Morales Demori R, Jain S, et al. Attenuation of the side effect profile of regadenoson: a randomized double-blinded placebo-controlled study with aminophylline in patients undergoing myocardial perfusion imaging. "The ASSUAGE trial." J Nucl Cardiol. 2012; 19: 448-457.

7 Iskandrian AE, Bateman TM, Belardinelli L, et al. Adenosine versus regadenoson comparative evaluation in myocardial perfusion imaging: results of the $A D$ VANCE phase 3 multicenter international trial. J Nucl Cardiol. 2007; 14: 645-658.

8 Nguyen KL, Bandettini WP, Shanbhag S, et al. Safety and tolerability of regadenoson CMR. Eur Heart J Cardiovasc Imaging. 2014; 15: 753-760.

9 Townsend R, Desai A, Rammelsberg D, et al. Safety and tolerability of intravenous regadenoson in healthy subjects: a randomized, repeat-dose, placebo-controlled study. J Nucl Cardiol. 2017; 24: 57-65.

10 Oleksiak A, Kruk M, Pugliese F, et al. Regadenoson dynamic computed tomography myocardial perfusion using low-dose protocol for evaluation of the ischemic burden. ULYSSES study. J Cardiovasc Comput Tomogr. 2020 Jan 21. [Epub ahead of print].

11 Cury RC, Kitt TM, Feaheny K, et al. A randomized, multicenter, multivendor study of myocardial perfusion imaging with regadenoson CT perfusion vs single photon emission CT. J Cardiovasc Comput Tomogr. 2015; 9: 103-112.

12 Baxa J, Hromádka M, Šedivý J, et al. Regadenoson-stress dynamic myocardial perfusion improves diagnostic performance of $\mathrm{CT}$ angiography in assessment of intermediate coronary artery stenosis in asymptomatic patients. Biomed Res Int. 2015; 2015: 105629

13 Bastarrika G, Ezponda A, Baizan AG, et al. Safety of regadenoson for vasodilation in cardiac MRI stress tests. Radiologia. 2020; 62: 213-221.
14 Doran JA, Sajjad W, Schneider MD, et al. Aminophylline and caffeine for reversal of adverse symptoms associated with regadenoson SPECT MPI. J Nucl Cardiol. 2017; 24: 1062-1070.

15 Page RL, Spurck P, Bainbridge JL, et al. Seizures associated with regadenoson: a case series. J Nucl Cardiol. 2012; 19: 389-391.

16 Agarwal V, DePuey EG. Regadenoson and seizures: a real clinical concern. J Nucl Cardiol. 2014 0ct; 21: 869-870.

17 Leaker BR, O'Connor B, Hansel TT, et al. Safety of regadenoson, an adenosine $A$ receptor agonist for myocardial perfusion imaging, in mild asthma and moderate asthma patients: a randomized, double blind, placebo-controlled trial. J Nucl Cardiol. 2008; 15: 329-336.

18 Thomas GS, Tammelin BR, Schiffman GL, et al. Safety of regadenoson, a se-

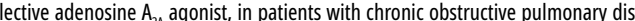
ease: a randomized, double-blind, placebo controlled trial (RegCOPD trial). J Nucl Cardiol. 2008; 15: 319-328.

19 Sokolska JM, von Spiczak J, Gotschy A, et al. Cardiac magnetic resonance imaging to detect ischemia in chronic coronary syndromes: state of the art. Kardiol Pol. 2019; 77: 1123-1133.

20 Zhao G, Linke A, Xu X, et al. Comparative profile of vasodilation by CVT-3146, a novel A2A receptor agonist, and adenosine in conscious dogs. J Pharmacol Exp Ther. 2003; 307: 182-189. 